\title{
Magnetic and Electric Field Effects on the Photoelectron Emission from Prototype LHC Beam Screen Material
}

\author{
V.V. Anashin', I.R. Collins², R.V. Dostovalov', N.V. Fedorov', O. Gröbner ${ }^{2}$, A.A. Krasnov', \\ O.B. Malyshev ${ }^{1}$ and E. E. Pyata ${ }^{1}$
}

\begin{abstract}
This paper describes experimental studies of the effect of a dipole field on the photoelectron emission and on the photon reflectivities from LHC beam screen material. These studies were performed using synchrotron radiation from the VEPP-2M storage ring at BINP (Novosibirsk). The particular surface roughness and geometry of the prototype LHC beam screen material requires dedicated experimental measurements. The experiments were performed under conditions close to those expected in the LHC. An important result obtained is that a dipole magnetic field attenuates the photoelectron emission from surface by more than two orders of magnitude with the magnetic field aligned parallel to the surface. The measurements of photon reflectivities, forward scattered and diffuse, and the azimuthal distribution of emitted photoelectrons from the same material are reported. These experimental results are important input for the final design of the LHC beam screen.
\end{abstract}

1 Members of the CERN-Russia Collaboration for the LHC; Budker Institute of Nuclear Physics, 630090 Novosibirsk, Russia 2 CERN, LHC Division

6th European Vacuum Conference (EVC-6)

7-12 December 1999, Villeurbanne, France

\author{
Administrative Secretariat \\ LHC Division \\ CERN \\ CH - 1211 Geneva 23 \\ Switzerland \\ Geneva, 15 March 2000
}




\title{
MAGNETIC AND ELECTRIC FIELD EFFECTS ON THE PHOTOELECTRON EMISSION FROM PROTOTYPE LHC BEAM SCREEN MATERIAL
}

\author{
V.V. Anashin ${ }^{\mathrm{a}}$, I.R. Collins ${ }^{\mathrm{b}}$, R.V. Dostovalov ${ }^{\mathrm{a}}$, N.V. Fedorov ${ }^{\mathrm{a}}$, O. Gröbner ${ }^{\mathrm{b}}$, A.A. Krasnov ${ }^{\mathrm{a}}$, \\ O.B. Malyshev and E. E. Pyata ${ }^{\mathrm{a}}$. \\ ${ }^{a}$ Budker Institute of Nuclear Physics, 630090 Novosibirsk, Russia \\ ${ }^{\mathrm{b}} \mathrm{CERN}, \mathrm{CH}-1211$ Geneva 23, Switzerland
}

\begin{abstract}
This paper describes experimental studies of the effect of a dipole field on the photoelectron emission and on the photon reflectivities from LHC beam screen material. These studies were performed using synchrotron radiation from the VEPP-2M storage ring at BINP (Novosibirsk). The particular surface roughness and geometry of the prototype LHC beam screen material requires dedicated experimental measurements. The experiments were performed under conditions close to those expected in the LHC. An important result obtained is that a dipole magnetic field attenuates the photoelectron emission from surface by more than two orders of magnitude with the magnetic field aligned parallel to the surface. The measurements of photon reflectivities, forward scattered and diffuse, and the azimuthal distribution of emitted photoelectrons from the same material are reported. These experimental results are important input for the final design of the LHC beam screen.
\end{abstract}

\section{INTRODUCTION}

The high flux of synchrotron radiation (SR) in the arc of the LHC, about $10^{17}$ photons/(s m), causes photoelectron emission from the walls of the beam screen. The emitted low energy electrons are accelerated in the field of the proton beam and may gain energies sufficient to create secondary electrons on the subsequent interaction with the beam screen, resulting in the creation of an electron cloud. The repeated recurrence of this process can result in beam-induced multipacting [1]. Theoretical simulations of the electron cloud in the LHC predict significant heat loads on the cryogenic system and electron stimulated gas desorption [2], [3], [4]. Experimental data are required as input to such simulations in order to estimate the influence of an electron cloud on the LHC operation. A large portion of the LHC beam vacuum system is located inside the dipole magnets. Previous studies have shown that the presence of the dipole field has a strong influence on the electron emission [5], [6]. In addition the heat loads in the dipole field are predicted to be strongly dependent on the azimuthal distribution of photons, determined by the photon reflectivity [2,3,4]. Experiments were performed to investigate the influence of a dipole field on the electron emission of various samples 
with different surface roughness [7] and to measure both the photon reflectivities and the effect of a dipole field from beam screen material with or without a saw-tooth surface structure [8]. These latter experiments were performed with SR at grazing incidence in order to simulate closely the geometry in the LHC. However, here the strength of the dipole field is significantly smaller than that in the LHC $(0.3 \mathrm{~T}$ versus $8.3 \mathrm{~T})$ and that the experiment was performed at room temperature and not at cryogenic temperatures. The saw-tooth structure has been proposed as an efficient method of reducing the forward scattered photon reflection in the LHC and hence adsorbing most of the photons on the equatorial plane of the beam screen.

The results on the influence of the dipole field on the photoelectron yield, performed with normal incident photons, are presented in section 2. In section 3 the results of the photoelectron yield and photon reflectivities from beam screen material with or without a saw-tooth surface structure, performed with grazing incident photons, are presented. A brief discussion and conclusions are made in section 4.

\section{Experiments performed with normal incident photons}

\subsection{Set-up and sample preparation}

The experimental set-up shown in Figure 1 was built on the beamline P2 of VEPP-2M to study the dependence of the photoelectron emission for photons at normal incidence as a function of a transverse dipole field (up to $0.6 \mathrm{~T}$ ). The sample was mounted on a sample holder, electrically isolated from the surrounding $46 \mathrm{~mm}$ diameter stainless steel chamber, and located in the centre of the magnetic field. An all-metal valve was used to isolate the vacuum of the experimental chamber and that of the beamline. The distance from the SR source to the collimator is about $11 \mathrm{~m}$. The sample, with vertical and horizontal dimensions of $44 \mathrm{~mm} \times 15 \mathrm{~mm}$, respectively is mounted on the sample holder with its surface perpendicular to the incident SR. The photon flux from VEPP-2M is collimated resulting in a $35 \mathrm{~mm} \times 10 \mathrm{~mm}$ area of the sample to be illuminated, i.e. the edges of the sample are not irradiated.

The increased vertical size of the photon beam and the sample was chosen to avoid attenuating the lower energy part of the spectrum. The sample was connected via an ammeter to a voltage supply $( \pm 300 \mathrm{~V})$. Hence, the potential on the sample could be controlled whilst the electron current from, or to, the sample could be measured. The magnetic field of 0 to $0.6 \mathrm{~T}$ was aligned approximately parallel to the sample surface and in the vertical direction. The experimental chamber has a reduced crosssection near the magnet centre to provide a stronger and more uniform magnetic field (see Figure 1). 


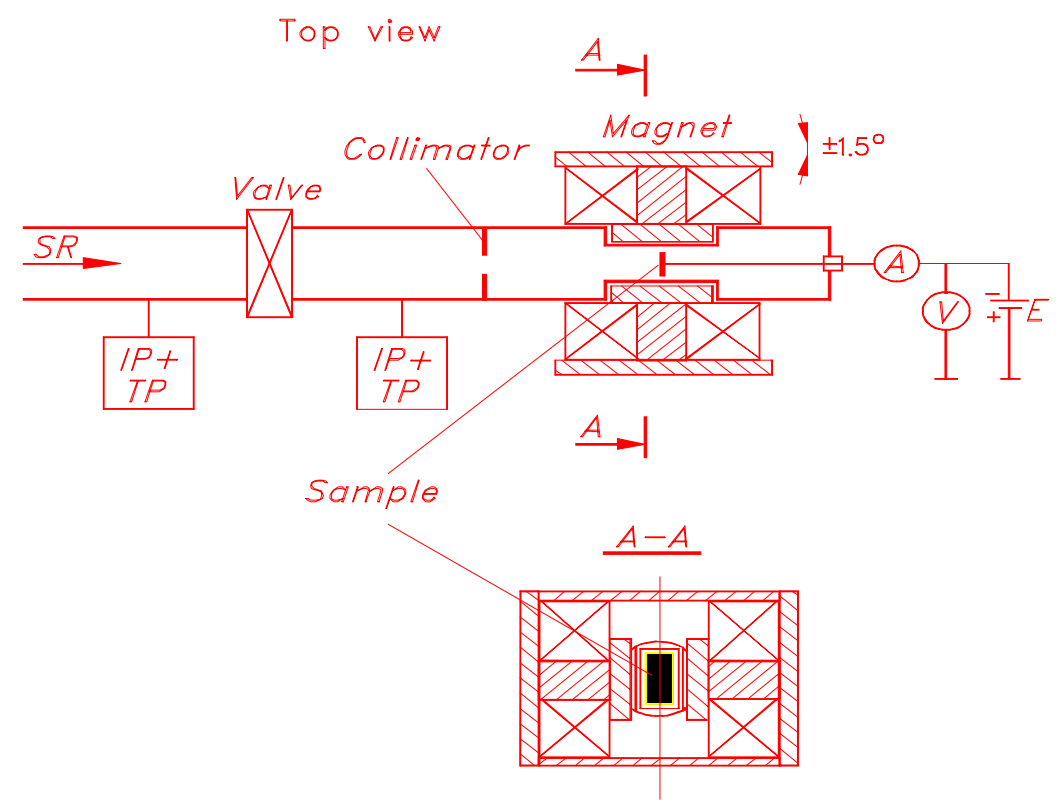

Figure 1: The layout of the installation.

Seven different samples were studied: a 316 series stainless steel, four made of copper colaminated stainless steel (the proposed material for the LHC beam screen), one machined from OFHC copper and a stainless steel electroplated with gold. The samples were degreased and cleaned with benzine and alcohol before installation.

Table 1: Definition and description of the samples studied.

\begin{tabular}{|c|c|}
\hline Sample name & Sample description \\
\hline SS & Stainless steel (316 series) sample made from a rolled sheet \\
\hline $\mathrm{Cu} / \mathrm{SS}-1$ & $\begin{array}{l}\text { Copper co-laminated stainless steel with the rolling direction perpendicular to the } \\
\text { magnetic field. }\end{array}$ \\
\hline $\mathrm{Cu} / \mathrm{SS}-2$ & $\begin{array}{l}\text { Copper co-laminated stainless steel with the rolling direction parallel to the } \\
\text { magnetic field. }\end{array}$ \\
\hline $\mathrm{Cu} / \mathrm{SS}-3$ & $\begin{array}{l}\text { Copper co-laminated stainless steel baked in air at } 300 \bullet \mathrm{C} \text { for } 5 \text { mins. with the } \\
\text { rolling direction parallel to the magnetic field. }\end{array}$ \\
\hline $\mathrm{Cu} / \mathrm{SS}-4$ & $\begin{array}{l}\text { Copper co-laminated stainless steel made with turned-in, long edges, i.e. } 5 \mathrm{~mm} \\
\text { wide strips at the long edges were turned to } 10-15^{\circ} \text { towards the SR with the rolling } \\
\text { direction parallel to the magnetic field. }\end{array}$ \\
\hline OFHC & $\begin{array}{l}\text { OFHC copper machined with longitudinal grooves aligned parallel to the magnetic } \\
\text { field. The grooves are } 1 \mathrm{~mm} \text { deep and } 3 \mathrm{~mm} \text { wide with a pitch of } 3.2 \mathrm{~mm} \text {. }\end{array}$ \\
\hline $\mathrm{Au} / \mathrm{SS}$ & Stainless steel electroplated with $6-\mu \mathrm{m}$ of gold \\
\hline
\end{tabular}




\subsection{Results}

In all experiments, the photoelectron yield was a measured parameter. It was estimated as a ratio of the measured output current $I_{m e s}$ (i.e. electron flux from or to the sample) to photon flux $\dot{\Gamma}$. Neither the effect of the beamline collimation nor the sample workfunction is taken into account in the estimation of the photoelectron yield, contrary to that in other studies [5].

$$
\kappa[\text { electrons / photon }]=\frac{I_{m e s}}{e \cdot \dot{\Gamma}}
$$

where $e$ is the electron charge.

The photoelectron yield was studied as a function of the different parameters:

1. Potential on the sample (or bias) from $-300 \mathrm{~V}$ to $+300 \mathrm{~V}$ at fixed dipole field between 0 and $0.6 \mathrm{~T}$;

2. Magnetic field from 0 up to $0.6 \mathrm{~T}$ at a fixed potential between 0 and $-300 \mathrm{~V}$.

3. Accumulated photon dose.

Table 2: Measured photoelectron yields, $\kappa$, of the various samples. The photon spectrum critical energy at which the measurements were made is also indicated.

\begin{tabular}{|l|c|c|c|c|}
\hline \multirow{2}{*}{ Sample } & Critical Energy & $\begin{array}{c}\text { Measurements with } \\
\mathrm{B}=0 \mathrm{~T} \text { and } \mathrm{U}=-300 \mathrm{~V}:\end{array}$ & \multicolumn{2}{|c|}{$\kappa(0.6 T) / \kappa(0 T)$} \\
\cline { 4 - 5 } & $\mathrm{E}_{\mathrm{c}}(\mathrm{eV})$ & $\kappa\left(\mathrm{e}^{-} / \gamma\right)$ & $\mathrm{U}=0$ & $\mathrm{U}=-300 \mathrm{~V}$ \\
\hline $\mathrm{SS}$ & 259 & 0.016 & 0.023 & 0.028 \\
\hline $\mathrm{Cu} / \mathrm{SS}-1$ & 253 & 0.015 & 0.010 & 0.029 \\
\hline $\mathrm{Cu} / \mathrm{SS}-2$ & 194 & 0.014 & 0.021 & 0.030 \\
\hline $\mathrm{Cu} / \mathrm{SS}-3$ & 112 & 0.014 & 0.020 & 0.015 \\
\hline $\mathrm{Cu} / \mathrm{SS}-4$ & 20 & 0.014 & 0.010 & 0.060 \\
\hline $\mathrm{Cu} / \mathrm{SS}-4$ & 319 & 0.018 & 0.024 & 0.080 \\
\hline $\mathrm{OFHC}$ & 102 & 0.008 & 0.028 & 0.013 \\
\hline $\mathrm{Au} / \mathrm{SS}$ & 356 & 0.027 & 0.042 \\
\hline
\end{tabular}

In general, the photoelectron yield was found to increase rapidly for increasing negative bias, corresponding to electrons leaving the sample, and saturates for a bias greater that $100 \mathrm{~V}$. It decreases rapidly with the dipole field when the magnetic field changes from 0 to about 0.1 to $0.2 \mathrm{~T}$, then decreases slowly, and is practically constant between 0.4 and $0.6 \mathrm{~T}$. Since this suppression at low fields can be attributed to electrons being constrained to move along the field lines and hence return to the strip from which they were emitted it is therefore thought unlikely that the yield will change for 
higher magnetic fields. The dependence of the photoelectron emission on the photon dose was found in comparing the data without the magnetic field at the beginning of the measurements. After a dose of $10^{22}$ photons the photoelectron emission yield reduces by about a factor of 2 . The main results are shown in Table 2.

\section{Experiments performed with grazing incident photons}

\subsection{Set-up and sample preparation}

The experimental set-up (shown in Figure 2), installed at the end of a second, short SR beam line, was located at a distance of about $1 \mathrm{~m}$ from the SR source point where the effect of the vertical collimation on the photon flux is negligible. Here the dimensions of the SR beam is defined by a set of horizontal $\left(\mathrm{C}_{\mathrm{h}}\right)$ and vertical $\left(\mathrm{C}_{\mathrm{v}}\right)$ collimators, $2 \mathrm{~mm}$ and $10 \mathrm{~mm}$ respectively, on entering the experimental system. The photon flux, through the collimators, was typically $10^{16}$ photons/s.

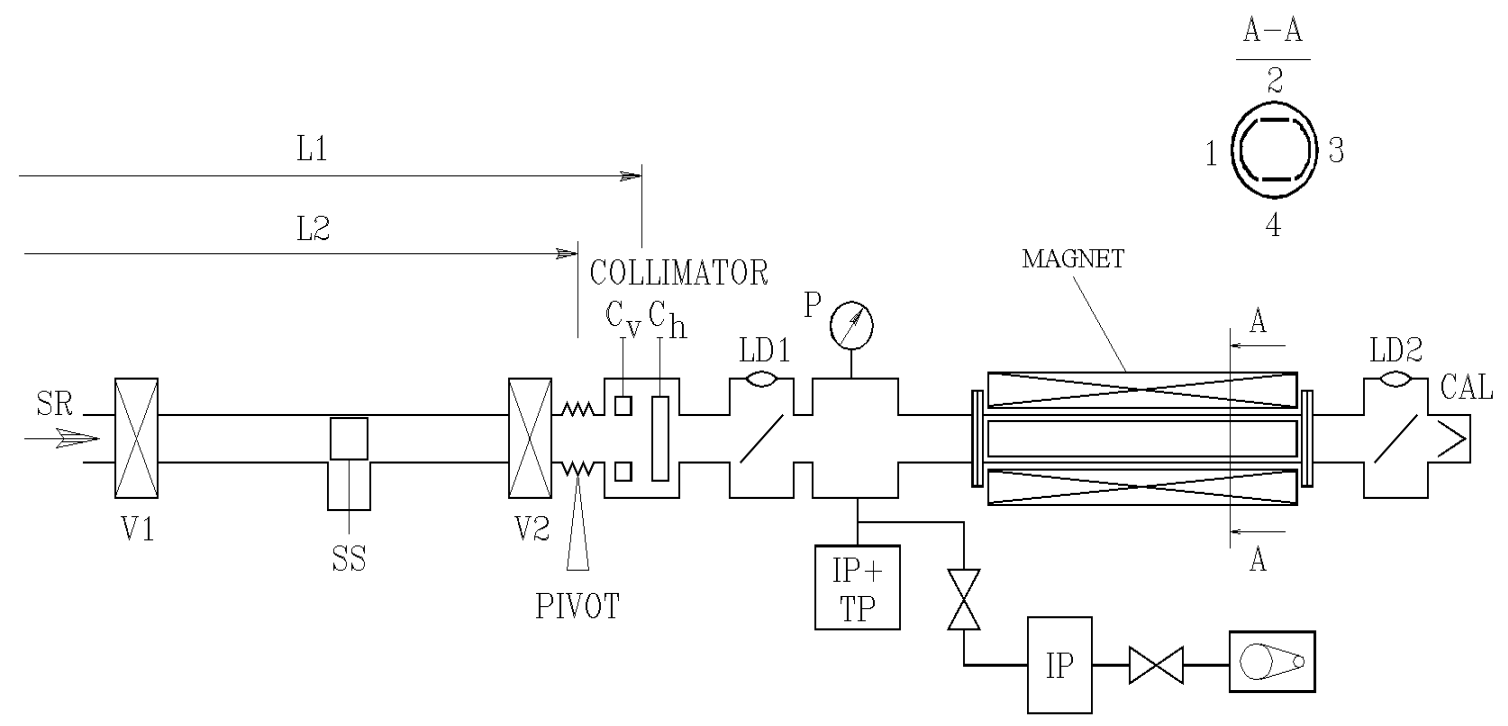

Figure 2: Set-up for measurements of the photon reflectivity and azimuthal photoelectron distribution in a magnetic field.

\subsection{Results}

The position of the photon beam with respect to the experimental system could be checked with two luminescent screens, (LD1) and (LD2). At the exit of the test system, a calorimeter (CAL) was installed to monitor the SR. This calorimeter was designed in such a way that it could either measure 
the total SR power or the total photon flux derived from the photoelectron current produced on the calorimeter. For the latter measurement the calorimeter was electrically biased with respect to the test chamber. A section of a prototype LHC beam screen, $34 \mathrm{~cm}$ in length, was cut into four strips: two rounded and two flat (see Figure 3). In a following experiment strip 1 was replaced with a flat strip of beam screen material with a saw-tooth structure on its surface. A dipole magnet with a field of up to $0.3 \mathrm{~T}$ was installed along the whole length of the vacuum chamber containing the strips. Strips 2 and 4 were perpendicular to magnetic field. The rounded strip 3 was opposite to strip 1 . The whole experimental system could be positioned either aligned straight or inclined at a given grazing angle with respect to the axis of the photon beam. In the straight position, the SR beam traversed the test system and was incident on the end calorimeter, while in the inclined position, the photon beam was incident at an angle of about $10 \mathrm{mrad}$ along strip 1 only reflected photons are able to reach the calorimeter.

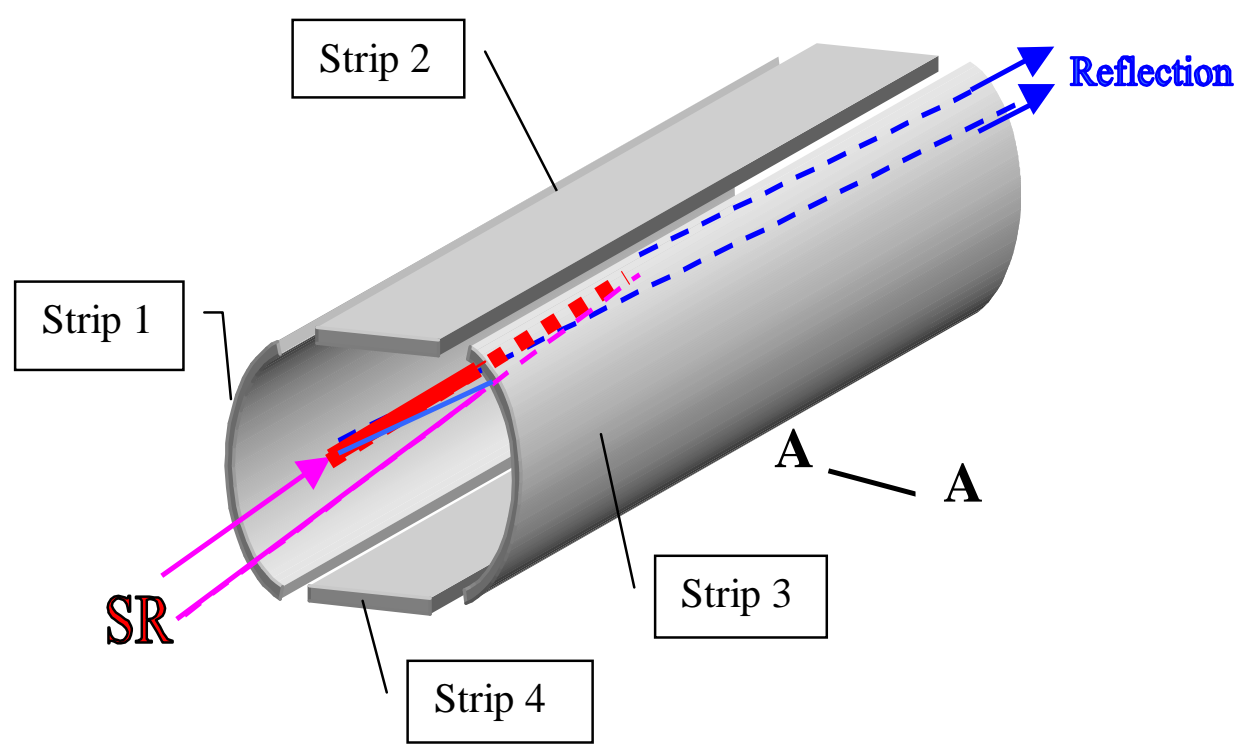

Figure 3: ample configuration for experiments of the photon reflectivity and azimuthal photoelectron distribution in a magnetic field.

During a typical measurement, the SR beam was incident on strip 1. The remaining strips received only reflected photons. Applying a negative bias in turn to each of the four strips while maintaining all others at the common ground potential, the photoelectron current produced by photons on the corresponding strip was measured. Assuming that the average photoelectric yield for the direct incident photons and for the reflected photons are not too different, it is possible to derive from the measured azimuthal distribution of the photoelectron signal the azimuthal distribution of the photons. The measurements were performed for a bias voltage from 0 to $290 \mathrm{~V}$ and with the magnetic field of $\mathrm{B}=0$ to $0.3 \mathrm{~T}$. 
Forward scattered reflection is defined as a ratio of measured values on the calorimeter, by current or power, in the two positions:

$$
\begin{aligned}
& R_{e}=I_{r e f} / I_{d i r} ; \\
& R_{W}=W_{r e f} / W_{d i r} .
\end{aligned}
$$

Diffuse photon reflectivity is defined as:

$$
R_{d i f}=\frac{I_{2}+I_{3}+I_{4}}{e \cdot \dot{\Gamma} \cdot Y}
$$

The yield is defined per adsorbed photon as:

$$
Y=\frac{\sum_{i=1}^{4}\left(I_{i} / e\right)}{\left(1-R_{e}\right) \dot{\Gamma}},
$$

where the currents $I_{i}$ from strips were measured at a bias of $-300 \mathrm{~V}$.

The main results are shown in Table 3. The adsorbed photon flux is that which is not reflected and $\mathrm{R}_{\mathrm{dif}} /\left(\mathrm{R}_{1}+\mathrm{R}_{\mathrm{dif}}\right)$ gives and indication of the importance of the diffusely reflected $\mathrm{SR}$, with respect to the

\begin{tabular}{|c|c|c|c|c|c|c|c|}
\hline \multirow{2}{*}{ Sample } & \multirow{2}{*}{$\begin{array}{l}\text { Critical } \\
\text { Energy } \\
E_{c}(e V)\end{array}$} & \multicolumn{2}{|c|}{$\begin{array}{c}\text { Forward Scattered } \\
\text { Reflection }\end{array}$} & \multirow{2}{*}{$\begin{array}{c}\text { Diffuse } \\
\text { Reflectivity } \\
R_{d i f}\end{array}$} & \multirow{2}{*}{$\begin{array}{c}\text { Photon } \\
\text { Adsorption } \\
R_{l}\end{array}$} & \multirow{2}{*}{$\frac{R_{d i f}}{R_{l}+R_{d i f}}$} & \multirow{2}{*}{$\begin{array}{c}\mathrm{Y} \\
\text { (electron/ } \\
\text { photon) }\end{array}$} \\
\hline & & $\begin{array}{l}R_{e} \text { (by } \\
\text { current) }\end{array}$ & $\begin{array}{l}R_{W} \text { (by } \\
\text { power) }\end{array}$ & & & & \\
\hline $\begin{array}{l}\text { Smooth } \\
\text { surface }\end{array}$ & 20 & 0.67 & - & 0.04 & 0.29 & 0.13 & 0.03 \\
\hline \multirow{2}{*}{$\begin{array}{l}\text { Saw-tooth } \\
\text { surface }\end{array}$} & 49 & 0.035 & - & 0.22 & 0.74 & 0.23 & 0.049 \\
\hline & 246 & 0.026 & 0.03 & 0.185 & 0.79 & 0.19 & 0.063 \\
\hline
\end{tabular}
direct irradiation, as a source of electrons in the vacuum chamber.

Table 3: A summary of the measured reflectivities and photoelectron yields per absorbed photon. The critical energy at which the measurements were made is also indicated.

The photoelectron current on strip 1 is attenuated by a factor of about 200 for the strip without saw-tooth in the dipole field above $0.2 \mathrm{~T}$ and about a factor of 5 for the strip with saw-tooth. The dipole field does not affect the photoelectron current from strips 2 and 4 , top and bottom. 


\section{Discussion and Conclusions}

The experiments with SR at normal incidence show that the different materials with the different surfaces have about the same photoelectron yield. It is found that the photoelectron current as a function of dipole field is attenuated by a factor of 30 to 50 reaching a saturation value at $0.4 \mathrm{~T}$.

The experiments performed at grazing incidence in a dipole field demonstrate that the electrons are constrained to move along the field lines, resulting in electrons emitted from strip 1 and strip 3 to return to the same strip from which they were created. In the case of the LHC these electrons interact weakly with the proton beam, gaining insufficient energy to cause multipacting or contribute a significant heat load [9]. On the other hand, photoelectrons from strips 2 and 4 (top and bottom) are not attenuated by the presence of the dipole field and thus they interact with the proton beam and contribute to the electron cloud.

The results presented here are important input to predict the behaviour of the electron cloud in the dipole fields of the LHC and to estimate electron stimulated gas desorption. The forward scattered reflectivity, as measured by the deposited power and by the photon flux, show significantly different results for the smooth surface and for the saw-tooth surface. With respect to the electron-cloud effect in the LHC a low reflectivity is desirable since it will limit the photoelectron production to the region of the primary impact of the photons in the equitorial plane where electrons are efficiently attenuated by the strong vertical dipole field [10]. From this point of view, the saw-tooth surface is attractive for the beam screen of the LHC.

\section{References}

[1] Gröbner, O., CERN LHC Project Report 127 (1997).

[2] Zimmermann, F., CERN LHC Project Report 95 (1997).

[3] Brüning, O.S., CERN LHC Project Report 158 (1997), Brüning, O.S., CERN LHC Project Report 190 (1997).

[4] Furman, M.A., CERN LHC Project Report 180 (1997).

[5] Baglin, V., Collins, I.R. and Gröbner, O., CERN LHC Project Report 206 (1998).

[6] Anashin, V.V., Malyshev, O.B., Fedorov, N.V., Nazmov, V.P., Goldenberg, B.G., Collins, I.R. and Gröbner, O., CERN LHC Project Report 266 (1999).

[7] Anashin, V.V., Dostovalov, R.V., Malyshev, O.B., Krasnov, A.A. and Pyata, E.E., CERN Vacuum Technical Note 99-03 (1999).

[8] Anashin, V.V., Malyshev, O.B., Fedorov, N.V. and Krasnov, A.A., CERN Vacuum Technical Note 99-06 (1999).

[9] Baglin, V., Collins, I.R., Gómez-Goñi, J., Gröbner, O., Henrist, B., Hilleret, N., Laurent, J-M., Pivi, M., Cimino, R., Anashin, V.V., Dostovalov, R.V., Fedorov, N.V., Malyshev, O.B., Krasnov, A.A. and Pyata, E.E., presented at $\mathrm{e}^{+} \mathrm{e}^{-}$Factories'99, KEK in Tsukuba, Japan $21^{\text {st }}-24^{\text {th }}$ Sept. 1999. [10] Baglin, V., Bruning, O., Calder, R., Caspers, F., Collins, I.R., Gröbner, O., Hilleret, N., Laurent, J-M., Morvillo, M., Pivi, M. and Ruggiero, F., EPAC-98, June 1998. 